\title{
El déficit lingüistico en personas con demencia de tipo alzhéimer: breve estado de la cuestión
}

\author{
José Luis PÉRez Mantero \\ Universitat de València \\ Facultad de Filología, Traducción y Comunicación \\ Avda. Blasco Ibáñez, 32 \\ 46010 Valencia \\ E-mail: jopemon4@alumni.uv.es \\ Tel. (+34) 9639864279
}

EL DÉFICIT LINGÜíSTICO EN PERSONAS CON DEMENCIA DE TIPO ALZHÉIMER: BREVE ESTADO DE LA CUESTIÓN

RESUMEN: Las personas con demencia de tipo alzhéimer sufren alteraciones lingüisticas. Se han llevado a cabo algunos estudios que describen la naturaleza de este déficit. Los resultados de estos trabajos demuestran que los componentes léxico-semántico y pragmático se encuentran mucho más deteriorados que los niveles fonético-fonológico y morfosintáctico del lenguaje. Nuestro propósito es elaborar un breve estado de la cuestión que nos permita tener una perspectiva global sobre el proceso de deterioro comunicativo. Además, enfatizamos la importancia del análisis lingüístico a la hora de diagnosticar la enfermedad de Alzheimer, ya que se pueden encontrar cambios en las habilidades discursivas desde la etapa prodrómica de la demencia. Finalmente, proponemos la creación de protocolos conversacionales que ayudarán a los hablantes con demencia de tipo alzhéimer y a sus interlocutores-clave a mantener una buena interacción comunicativa durante el mayor tiempo posible.

PALABRAS CLAVES: demencia; alzhéimer; lingüística clínica; déficit lingüístico; comunicativas. habilidades

SUMARIO: $1 . \quad$ Introducción: lenguaje y demencia. 2. Cuestiones metodológicas. 3. Las Demencias de tipo alzhéimer. 4. Conclusiones.
LINGUISTIC IMPAIRMENTS IN SUBJECTS WITH DEMENTIA OF THE AlzheIMER's TYPE: STATUS OF THE ISSUE

ABSTRACT: Linguistic abilities are impaired in subjects with dementia of the Alzheimer's type. Some studies have been carried out in order to describe the nature of this deficit. The results of these studies have shown that the lexical-semantic and pragmatic components are much more damaged than phonetical, phonological morphosyntactical aspects of language. Our intention is to elaborate a brief status of the issue which permits us to have a global outlook of the communicative deterioration process. It is also emphasized the importance of linguistic analysis when it comes to diagnosing Alzheimer's disease since discourse changes can be found in the preclinical stage of dementia. Finally, we propose the creation of conversational protocols that will help subjects with dementia of Alzheimer's type and their key conversational partners to preserve good communicative interactions for as long as possible.

KEY WORDS: dementia; alzheimer; clinical linguistics; linguistic impairments; communicative abilities.

SUMMARY: 1 . Introduction: language and dementia. 2. Methodological issues. 3. Dementia of the Alzheimer's type. 4. Conclusion.
LE DEFICIT LINGUISTIQUE EN PERSONNES AVEC DEMENCE DE TYPE AlZheimer: BREF ETAT DE LA QUESTION

RÉSUMÉ: Les capacités linguistiques de sujets atteints de démence comme l'Alzheimer se détériorent au fil du temps. Certaines études on été faites pour décrire la nature de ce déficit. Les résultats de ces études on montrés que les composants lexico-sémantique et pragmatique sont beaucoup plus touchés que les aspects phonétiques, phonologiques morphosyntaxiques du langage. Notre but est d'élaborer une liste de tous les aspects qu'ils peuvent nous permettre d'avoir un regard global sur le processus de détérioration de la communication. L'importance de l'analyse linguistique quand il s'agit de diagnostiques la maladie d'Alzheimer est aussi mise en avant, étant donné que les difficultés de langage peuvent survenir avant le stade clinique de la maladie. Enfin, nous proposons la création d'exercices de conversation pour aider le patient et leur entourage à préserver une bonne communication pour aussi longtemps que possible.

MOTS CLÉS: démence; alzheimer; linguistique clinique; déficit linguistique; habilités communicatives.

SOMMAIRE: 1 . Introduction : langage et démence. 2 . Questions méthodologiques. 3. Les Démences de type Alzheimer. 4. Conclusions. 


\section{E1 déficit lingüistico en personas con demencia de tipo alzhéimer: breve estado de la cuestión}

JoSÉ LUIS PÉREZ MANTERO

\section{INTRODUCCIÓN: LENGUAJE Y DEMENCIA}

La investigación de los déficits lingüísticos es una de las áreas de estudio más recientes de la Lingüística aplicada, cuyo desarrollo a través de la Lingüística clínica ha atendido ya a una amplia lista de situaciones patológicas con incidencia en la capacidad lingüistica de las personas que las sufren. En este artículo pretendemos recoger las principales aportaciones que desde ámbitos tan alejados como la Neurología, la Psicología o la Lingüística han contribuido a describir las alteraciones lingüísticas en personas con demencias de tipo alzhéimer (DTA).

Este tipo de demencia neurodegenerativa no afecta únicamente a la memoria -como normalmente se cree-, sino que produce el desmantelamiento progresivo de todas las funciones cognitivas superiores, entre las que se encuentra el lenguaje. Así, la importancia de la investigación lingüística en personas con DTA reside en varios puntos. En primer lugar, el análisis de las habilidades lingüisticas podría ser clave para contribuir a diagnosticar la enfermedad de Alzheimer de forma temprana (actualmente el diagnóstico está cambiando y no se basa solo en pruebas clínicas, sino que presta cada vez mayor atención a la búsqueda de marcadores en pruebas complementarias). En segundo lugar, la descripción del deterioro lingüístico puede conducirnos a diferenciar distintas etapas de la demencia y a comprender mejor las alteraciones cerebrales que provoca la enfermedad. Finalmente, el análisis del lenguaje de las personas con DTA nos permitiría crear protocolos de intervención logopédica conversacional entre el hablante enfermo y sus interlocutoresclave, específicamente diseñados para esta situación. Esto ayudaría, sin duda, a que las personas demenciadas pudieran mantener su capacidad de comunicación hasta un punto más avanzado de la enfermedad, mejorando así su calidad de vida.

En la primera parte de nuestro trabajo desgranamos algunas cuestiones referidas a la metodología de investigación seguida por los diversos estudios sobre lenguaje y demencia que presentaremos más tarde. A continuación, una vez hayamos definido las características principales de las demencias, estableceremos una división de la enfermedad por etapas dependiendo de las alteraciones lingüísticas. Por último, analizaremos los déficits comunicativos que aparecen a causa de una DTA a partir de los distintos componentes y destrezas del lenguaje. 


\section{Cuestiones metodológicas}

Los modelos teóricos bajo los cuales se lleva a cabo cualquier investigación han tenido y todavía tienen una gran importancia en los estudios sobre patologías del lenguaje (Gallardo Paúls, 2006). Así, hasta la eclosión del enfoque comunicativo en la Lingüística, las investigaciones sobre el déficit lingüístico seguían predominantemente modelos teóricos de tipo generativista, especialmente los de raigambre psicológica o neuropsicológica, lo que tuvo claras consecuencias en el desarrollo de disciplinas como la Neurolingüística, la Psicolingüística o la misma Lingüística Clínica. Por ejemplo, el hecho de que se asumiera la identificación entre gramática y lenguaje supuso una concepción estrecha de la Lingüística y de las alteraciones lingüisticas que favorecía el estudio de aquellas patologias, fundamentalmente la afasia, que conllevaban alteraciones en los componentes fonético-fonológico, morfosintáctico y/o léxico-semántico del lenguaje, minimizando el interés por los déficits de alcance pragmático, más propios de situaciones como el Síndrome de Williams, el Síndrome de Asperger o las DTA.

La adopción de una perspectiva pragmática en la Lingüística desde los años 80 ha permitido, además, detectar que los tests tradicionales examinaban el lenguaje potencial del hablante y no el uso lingüístico real. Por ejemplo, mientras algunos afásicos puntuaban muy bajo en los tests gramaticales, sus habilidades pragmáticas les permitian comunicarse; paralelamente, los hablantes con DTA pueden conservar la gramática casi intacta, pero muestran serios déficits de competencia pragmática que dificultan la comunicación. El fallo de estos tests se debe, fundamentalmente, a que las pruebas prescinden generalmente del contexto en el que se sitúa toda comunicación humana:

\footnotetext{
El empleo de las baterías tradicionales encubre las dificultades que experimentan estos pacientes para utilizar el lenguaje en contextos interactivos y comunicativos, ya que estas pruebas miden habilidades lingüisticas de forma aislada, y raramente aparecen como tal[es] en el contexto comunicativo de la vida diaria (Muñoz y Melle, 2004: 854).
}

Además, si repasamos el grueso de los estudios existentes sobre alteraciones lingüísticas en personas con DTA, llegamos a la conclusión de que la gran mayoria de los trabajos que solo analizan las habilidades gramaticales utilizan baterias de tests elaboradas originalmente para diagnosticar casos de afasia (cfr. Appell et al., 1982; Murdoch et al., 1987; Horner et al., 1992; Blair et al., 2007), sin tener en cuenta las características distintivas de ambas enfermedades. Por un lado, atendiendo al modo en que se ocasiona el daño, la diferencia estriba en que las patologías focales, como la afasia, afectan predominantemente a las funciones cognitivas asociadas a las zonas cerebrales que han sufrido la lesión (y a algunas que se puedan ver contagiadas por contigüidad), 
mientras que en un proceso degenerativo las funciones cognitivas se desmontan a partir de la pérdida de conexiones entre neuronas situadas en puntos diversos del cerebro, por lo que las habilidades afectadas variarán de acuerdo a cómo progrese el deterioro neuronal. Por otro lado, las afasias suponen una alteración de la capacidad lingüística casi únicamente; el sujeto afásico no muestra alteraciones destacables en otras funciones intelectuales, más allá de las limitaciones derivadas del déficit verbal. Sin embargo, en las demencias, el déficit lingüístico viene provocado en gran medida por el deterioro de otras funciones, como la memoria, la concentración o la capacidad de razonamiento. Esta es la causa de que los sujetos con DTA puntúen extraordinariamente bajo en pruebas que miden habilidades sintácticas y léxico-semánticas (Emery, 1985; Bates et al., 1995; Groves et al., 2004).

Es cierto, no obstante, que actualmente contamos con un número creciente de trabajos basados en datos extraidos de corpus de conversaciones o, en su defecto, de narraciones. Entre los primeros destaca el estudio pionero de Hamilton (1994) en el que esta autora presenta una investigación de tipo longitudinal a partir de catorce conversaciones mantenidas con una informante con DTA durante cinco años. Este trabajo condujo a la realización de otros (Rhys y SchmidtRenfree, 2000; Maclagan y Mason, 2005), si bien no conocemos un estudio que englobe tantos aspectos como el de Hamilton. Más comunes han sido los trabajos que recogían narraciones de vida (Ramanathan, 1997) y relatos de cuentos tradicionales (Labos, 2002).

Pues bien, una vez hemos comentado los principales métodos de investigación en Lingüística clínica, con una mención especial a algunos estudios sobre lenguaje y demencia, nos iremos aproximando al meollo de nuestro propósito inicial: realizar un repaso crítico por los trabajos que han investigado las habilidades lingüisticas de las personas con DTA. Para ello, comenzaremos detallando las características de la enfermedad y, finalmente, expondremos de una manera más profunda los déficits lingüísticos asociados.

\section{LAS DEMENCIAS DE TIPO ALZHÉIMER}

\subsection{CARACTERÍsticas y diagnóstico de la ENFERMEdAd}

La demencia, en general, puede definirse como "un deterioro adquirido de varias funciones cognitivas, no necesariamente permanente ni progresivo, y de una importancia tal que impide al paciente el correcto desenvolvimiento de sus actividades sociolaborales" (Robles Bayón y Vilariño Vilariño, 1998: 73). Esta sucinta caracterización de la demencia nos descubre ciertos aspectos relacionados con la enfermedad que debemos aclarar. En primer lugar, el hecho de que sea un deterioro "adquirido" nos indica algo ya conocido: las demencias se producen en una 
edad adulta o en la vejez, cuando las funciones intelectuales ya se han desarrollado. En segundo lugar, estas funciones mentales o cognitivas que se ven alteradas en la demencia pueden serlo en un grado variable dependiendo del tipo de demencia o de la gravedad de la misma. Como recogen Robles Bayón y Vilariño Vilariño (1998: 73), las funciones cognitivas comprenden fundamentalmente "la memoria, la gnosis, el lenguaje, el cálculo, la praxia ideomotriz, la habilidad visuoconstructiva o visuomotora, el pensamiento abstracto y la capacidad de razonamiento". De todas ellas, por supuesto, nos centraremos en el lenguaje, aunque sin dejar de lado otras funciones (la memoria, la habilidad visuomotora, la capacidad de razonamiento) que afectan tanto a la capacidad comunicativa del hablante como a los resultados que obtienen en los tests de habilidades lingüísticas. Por último, en la definición de demencia se afirma que esta no es un trastorno necesariamente progresivo, ya que existen algunos tipos de demencia (las subcorticales, por oposición a las de tipo alzhéimer) que normalmente no se agravan con el paso del tiempo.

En cuanto a la etiología de la enfermedad, a lo largo de las últimas décadas se han propuesto multitud de causas, ya que ninguna ha demostrado ser el origen indiscutible de la demencia. No obstante, sí parece cierto que varias de estas causas confluyen en su aparición; por esta razón, podemos considerar la demencia como un "sindrome clínico plurietiológico" (Alonso, et al., 2004: 6). Centrándonos en la DTA, cabe recordar que todos los procesos degenerativos tienen un fondo genético, donde determinados genes están alterados a causa de una mutación en el propio enfermo o por culpa de un proceso hereditario.

No obstante, esta no es la única hipótesis que se ha esgrimido como válida para caracterizar la etiología de las demencias. Algunos autores hablan de microtraumatismos repetitivos o de la exposición a sustancias tóxicas como los posibles motivos de la aparición de la demencia. Sin embargo, las causas que normalmente se aducen para explicar este proceso degenerativo son la pérdida de neuronas, sobre todo, en las zonas del hipocampo y de la corteza cerebral (lo que provocaría la pérdida de memoria y el deterioro de las funciones cognitivas) y la presencia de un número muy elevado de placas neuríticas o seniles que no se corresponde con la edad del paciente (Murillo, 1999).

Dejando de lado los aspectos neurológicos y anatómicos de la enfermedad, el diagnóstico de la demencia suele hacerse a través de tests neuropsicológicos que evalúan el deterioro cognitivo de los pacientes. Algunas de las pruebas más utilizadas son el Mini Mental State Examination (Folstein et al., 1975) y la Alzheimer's Disease Assessment Scale-Cognitive (Rosen, et al., 1984). Sin embargo, solo incluyen unos pocos ítems relacionados con el lenguaje y la comunicación. Por el contrario, otras escalas, como el Functional Linguistic Communication Inventory (Bayles y Tomoeda, 1996) y la Severe Impairment Battery Language Scale (Ferris et al., 2009), están específicamente diseñadas para 
analizar el lenguaje a partir de una serie de categorias: escritura, lectura, comprensión, fluidez verbal, denominación, repetición y discurso.

De hecho, el examen de las habilidades comunicativas ha demostrado ser muy importante para diagnosticar la aparición de una DTA, ya que en el estado inicial de la enfermedad, esta es dificilmente distinguible de un proceso de envejecimiento normal, caracterizado por una mengua en muchas de las funciones cognitivas anteriormente mencionadas. Algunos estudios indican que las alteraciones discursivas pueden ser uno de los primeros signos de la DTA, a menudo identificables años antes de que el déficit de memoria se haga evidente. Por ejemplo, Schröder et al. (2010) compararon la producción discursiva de un grupo de personas que años más tarde desarrollarian una DTA con la de otro grupo que tuvo una vejez normal. Se constató que ya en la edad adulta las narraciones de ambos grupos diferian en cuanto al contenido proposicional y a la complejidad gramatical, siendo menor en los textos de los individuos a los que posteriormente se les diagnosticaria una DTA. En concreto, parece ser que el lenguaje de las personas que se encuentran en la fase preclínica de la enfermedad se caracteriza por una menor informatividad, lo que se traduciría en la producción de enunciados incompletos, incoherentes y repeticiones de lo ya dicho (Murray, 2010).

\subsection{RASGOS LINGÜÍSTICOS DE LAS DTA}

En general, los estudios clínicos nos muestran que las alteraciones en las DTA se sitúan en el componente semántico y en el componente pragmático del lenguaje, al tiempo que los niveles fonético-fonológico y morfosintáctico se encuentran relativamente preservados hasta la última fase de la enfermedad. Como bien expresan Appell et al. (1982: 83), el lenguaje espontáneo de los sujetos con alzhéimer se caracteriza de la siguiente manera:

Fluent irrelevant speech, with well-preserved syntax and words, yet for practical purposes the meaning is lost. In certain cases, some referential relationship can be established, but this is rarely in response to the question asked or the topic discussed. Nevertheless, the sentences are often complete and more or less correct grammatically, without any phonemic disturbances or articulatory problems.

Esto ocurre, básicamente, porque las habilidades pragmáticas y semánticas requieren procesos más complejos que dependen de otras funciones cognitivas deterioradas, como la memoria o la capacidad de planificación y razonamiento. En cambio, no se registran problemas en relación con la articulación de palabras ni con el reconocimiento de un fonema determinado. Lo mismo ocurre con la concordancia gramatical, el orden de palabras y otros aspectos propios del nivel morfosintáctico. El motivo es que estos componentes lingüísticos están radicados en áreas 
temporales y frontales del hemisferio cerebral dominante, normalmente el izquierdo, y la DTA afecta a las zonas posteriores de la corteza cerebral.

Las investigaciones sobre el déficit lingüístico en las DTA han alternado un estudio por componentes y por destrezas semióticas (expresión y comprensión orales, denominación, repetición, lectura, escritura) ${ }^{1}$, centrándose en un tipo o en otro. A nuestro modo de ver, la naturaleza de las alteraciones lingüísticas en la demencia nos permite organizar su análisis, en primer lugar, a partir de los cuatro componentes en los que podemos dividir el estudio lingüístico: fonético-fonológico, morfosintáctico, léxico-semántico y pragmático. Dentro de esta clasificación por componentes podemos dedicar el espacio necesario a cada una de las destrezas semióticas mencionadas, dotando, además, de un lugar diferenciado a la descripción de las habilidades para la repetición y la lectoescritura.

Sin embargo, al ser la DTA una patología de carácter progresivo, algo que se manifiesta claramente en la evolución de las habilidades comunicativas, el estudio de las alteraciones lingüisticas indefectiblemente tiene que tomar un cariz temporal por el cual descubramos el proceso de deterioro del lenguaje en estos individuos. Así, a partir de los estudios realizados en informantes que se situaban en distintos estadios de la enfermedad, Bayles (1984) y Obler y Albert (1984) proponen tres fases en cuanto al deterioro comunicativo ${ }^{2}$ :

1) La etapa inicial suele durar entre dos y cinco años. Las primeras dificultades lingüísticas pueden tardar en aparecer algún tiempo, ya que normalmente vienen precedidas por alteraciones de otras funciones cognitivas, como la memoria episódica. Entre estas alteraciones destacan los problemas de denominación y acceso a determinada palabra (anomia); el lenguaje se vuelve impreciso y, a consecuencia de ello, puede aparecer una ligera verbosidad o logorrea, ya que son conscientes de su dificultad expresiva; la comprensión se encuentra bastante preservada, al igual que ocurre con la sintaxis; en cuanto a las habilidades pragmáticas, se describe generalmente una disminución en la iniciativa del habla y una dificultad creciente para detectar recursos humorísticos e irónicos.

\footnotetext{
${ }^{1}$ La bibliografia sobre las alteraciones del lenguaje, quizás basándose en la clásica distinción entre afasia de Broca y afasia de Wernicke, ha intentado dividir el estudio del lenguaje en una serie de destrezas semióticas que se estudiaban de forma separada, como si se tratara de procesos que no tienen relación entre ellos. Lo mismo podemos decir del estudio por componentes.

2 Algunos autores (Murillo, 1999), en cambio, consideran una cuarta fase desgajada de la tercera, el "estadio o etapa terminal", que duraría aproximadamente un año a partir de la afectación del lóbulo temporal izquierdo. Se caracteriza por un estado de mutismo total. Otros autores (Macoir y Turgeon, 2006) añaden una etapa prodrómica o preclínica en la que se observan leves casos de anomia.
} 
2) La etapa moderada puede durar entre tres y cinco años, aproximadamente. Todos los déficits comunicativos presentes en la fase anterior se agravan en esta etapa: las alteraciones en el componente semántico y la dificultad para recuperar la palabra adecuada al contexto provocan que el individuo con DTA cree numerosos neologismos, una situación que puede hacerse grave hasta el punto de convertir su discurso en una jerga; otra consecuencia de este déficit son las abundantes parafasias semánticas (sustitución de un término por otro de significado similar o relacionado). Las construcciones sintácticas suelen ser más breves y se caracterizan por la presencia de un buen número de circunloquios motivados por la consabida dificultad para encontrar las palabras que se buscan. Por esta razón, el discurso normalmente se considera vacío de significado e incoherente. Los problemas de comprensión verbal se hacen más evidentes en esta fase de la enfermedad. Sin embargo, la repetición es una habilidad que se conserva hasta un estado muy avanzado de la demencia. Finalmente, en relación a la lectoescritura, parece ser que existen errores al escribir (agrafias) debidos a la adición, sustitución y omisión de letras; la capacidad para leer en voz alta, por su parte, no se encuentra muy alterada, aunque esto no implica que comprendan todo lo que leen.

3) La etapa avanzada tiene una extensión muy variable en el tiempo. En esta fase, la persona con DTA se instala en una situación de apatía que resulta en un notable decrecimiento del lenguaje espontáneo. Los errores de expresión se hacen muy frecuentes: la anomia está generalizada y llegan a aparecer parafasias fonológicas. Si en etapas anteriores la única destreza expresiva bien conservada era la repetición, en esta fase la situación deriva en un tipo de discurso repleto de ecolalias (repetición involuntaria del discurso ajeno) y glosomanías (desarrollo exclusivo de los temas de conversación preferidos). La sintaxis también se ve afectada por esta pobreza expresiva, dando lugar a multitud de estereotipias (repetición involuntaria de cierta unidad lingüística, ya sea un sintagma, un neologismo, etc.) La comprensión se ve igualmente alterada, llegándose a un punto en el que la persona demenciada no atiende a órdenes simples, algo que por otra parte puede ser debido a la apatía o a la dificultad para participar en una conversación de forma ágil. En referencia a las habilidades pragmáticas, el hablante apenas muestra iniciativa comunicativa ni contesta la mayoría de las veces a las preguntas que se le formulan, por lo que podemos decir que el deterioro comunicativo es muy importante en este punto. Finalmente, el hablante cae en un estado de mutismo.

Una vez hemos hecho un repaso por la evolución de las alteraciones lingüisticas presentes en la enfermedad de Alzheimer, punto necesario para entender el deterioro de la capacidad comunicativa que se da en estos sujetos, nos centraremos en cada uno de los componentes lingüísticos y de 
las destrezas semióticas que pueden verse afectados por la demencia. Para ello, tomaremos como punto de partida los estudios existentes en esta materia, tanto si se dedican a un aspecto concreto del lenguaje como si buscan hacer una descripción global de la comunicación de este grupo de población.

\subsubsection{EL DÉFICIT FONÉTICO-FONOLÓGICO}

A priori, ni la articulación ni la discriminación de fonemas son un problema que debamos adscribir a las alteraciones lingüísticas que surgen en una DTA. Por esta razón, los estudios encaminados a analizar las manifestaciones lingüísticas en esta patología han prestado poca o nula atención al componente fonético-fonológico. No obstante, algunos autores sí mostraron interés por examinar estos aspectos, motivados sin duda por los déficits de este tipo presentes en las afasias.

Asi, utilizando baterias de tests estandarizadas para casos de afasia, Price et al. (1993) encontraron que los sujetos con DTA no se ajustaban a los perfiles de actuación de los afásicos en cuanto a las alteraciones articulatorias. Murdoch et al. (1987) se suman a considerar que existe una disociación en los sujetos con DTA entre los componentes fonológico y sintáctico, por un lado, y los componentes semántico y pragmático, por otro, ya que las personas demenciadas eran capaces de corregir errores de fonología y de sintaxis incluso en un estadio avanzado del deterioro cognitivo, algo que no conseguian hacer cuando se trataba de un error de tipo semántico. Únicamente se han encontrado casos de alteraciones fonológicas en tareas que piden repetir frases o pseudopalabras en contextos experimentales (Glosser et al., 1997). Estas son pruebas cuyo contenido no se asemeja a la comunicación coloquial y que, además, requieren de una buena memoria a corto plazo.

No obstante, como sabemos, en la etapa avanzada de la demencia suelen aparecer parafasias fonológicas (omisiones, sustituciones o intercambio en la secuencia de fonemas de una palabra). Así, la mayoría de los trabajos existentes recoge el hecho de que hasta esta fase, en la que el deterioro lingüístico es muy acusado, no se presentan este tipo de alteraciones ${ }^{3}$.

\subsubsection{EL DÉFICIT MORFOSINTÁCTICO}

\footnotetext{
${ }^{3}$ En un estudio más reciente, Croot et al. (2000) describen una serie de individuos con DTA que muestran alteraciones fonéticas y fonológicas en una etapa temprana de la enfermedad: falsos comienzos, parafasias fonológicas y una capacidad articulatoria reducida. No obstante, reconocen su extrañeza ante estos hechos y nos informan que los sujetos estudiados presentaban lesiones en las áreas perisilvianas de la corteza cerebral, lo que nos puede llevar a pensar que en realidad no estaban tratando con pacientes de alzhéimer que tuvieran una evolución extraña del deterioro lingüístico, sino que eran personas con un tipo distinto de demencia, la demencia frontotemporal.
} 
Las habilidades gramaticales se encuentran preservadas en la etapa inicial y moderada de la demencia. De acuerdo con la hipótesis predominante en los estudios de Lingüística Clínica, Appell et al. (1982) confirmaron la buena actuación sintáctica de los individuos con DTA tras ser sometidos a pruebas que tenian en cuenta aspectos como la longitud y la estructura de las oraciones o el uso de conectores extraoracionales, entre otros.

La gran mayoría de los trabajos que han evaluado la competencia sintáctica de estos individuos se ha centrado en la producción de estructuras sintácticas y no en su comprensión. Al ser la DTA una patología lingüística de tipo fluente, es presumible que las personas que la padecen no tengan excesivos problemas con la expresión. Sin embargo, esto podría ser diferente en el plano de la comprensión. En un estudio de Emery (1985) en el que se emplean el Test for Sintactic Complexity y el Token Test los resultados fueron abrumadores a favor de que la comprensión de la sintaxis se encontraba muy alterada. Sin embargo, lo que Emery no tuvo en cuenta es que estos tests requieren habilidades visuoespaciales, praxis, memoria y competencia semántica para ser completados satisfactoriamente. Posteriormente, Rochon, et al. (1994) observaron que las mayores dificultades se presentaban cuando las oraciones constaban de varias proposiciones y en los casos en los que todos los papeles temáticos posibles estaban cubiertos. No obstante, en casi el $85 \%$ de las ocasiones las oraciones fueron entendidas correctamente. De todos modos, debido al deterioro cognitivo y, especialmente, de la memoria, la sintaxis se va simplificando a lo largo de las distintas etapas de la demencia (Chapman y Ulatowska, 1997) hasta que la fluidez verbal es tan reducida que impide toda comunicación por parte del sujeto con DTA.

\subsubsection{EL DÉFICIT LÉXICO-SEMÁNTICO}

Al contrario de lo que ocurre con el componente fonético-fonológico y el componente morfosintáctico, el nivel léxico-semántico está claramente afectado en las demencias corticales posteriores. De hecho, los primeros síntomas de tipo lingüístico que anuncian la existencia de la enfermedad de Alzheimer se relacionan con una disminución del vocabulario y una dificultad para recuperar la palabra buscada. La pronta y frecuente aparición de estas alteraciones lingüísticas ha favorecido la realización de numerosos estudios al respecto (Martin y Fedio, 1983; Huff et al., 1986; Bayles-y Tomoeda, 1990; Smith et al., 1995; Forbes-Mckay y Venneri, 2005).

El primer aspecto que debemos aclarar es el de la verdadera naturaleza del déficit, dejando de lado, en un primer momento, las diferentes formas a través de las cuales los hablantes tratan de compensar ese déficit. De acuerdo con los diferentes modelos psicolingüísticos que tratan de explicar 
el proceso de la expresión lingüística, existirian tres niveles (semántico, léxico y fonológico) en la producción oral ${ }^{4}$. En el nivel semántico se encontrarian representados los conceptos, pero no como unidades, sino como el conjunto de rasgos -semas- que caracterizan a cada concepto; en el nivel léxico se localizan las representaciones de las palabras, y en el nivel fonológico está representado cada uno de los fonemas de una lengua (Cuetos, 2003).

De acuerdo con esta división por niveles, las posibles alteraciones en cada uno de ellos puede ocasionar un tipo diferente de anomia; es decir, la anomia semántica será el resultado de un fallo en el proceso de activación conceptual, la anomia léxica estará provocada por un error en el acceso a la palabra y la anomia fonológica será la consecuencia de no poder recuperar los fonemas que constituyen una palabra. Los resultados de los tests de denominación, fluencia verbal y categorización semántica sugieren que existe un deterioro del conocimiento semántico en lugar de un déficit en el acceso léxico 5 (Nakamura et al., 2000; Paganelli et al., 2003).

A continuación nos centraremos en los efectos que este déficit provoca en el componente léxico-semántico y en el lenguaje de las personas con demencia, en general. Los primeros problemas de denominación y de fluidez verbal se manifiestan en la utilización excesiva de "palabras ómnibus", como cosa o lugar. Esta situación, que se agrava con el tiempo, es una de las causas de que el lenguaje de las personas con DTA sea caracterizado como vago o impreciso. Otra de las claves que nos señalan el déficit semántico de un sujeto es su utilización de palabras relacionadas, pero no estrictamente sinónimas, en lugar de la palabra adecuada. Normalmente, estas sustituciones se dan entre palabras que pertenecen al mismo campo semántico (decir bicicleta en lugar de motocicleta) o que están conectadas en la mente del hablante por alguna razón (decir circo cuando se refiere a un león). Estas sustituciones de una palabra por otra se denominan "parafasias semánticas". En el estudio de Huff et al. (1986), estos investigadores comprobaron que los sujetos con DTA podian nombrar bastantes objetos a partir de dibujos, pero que en una tarea de nombrar el máximo de elementos posibles pertenecientes a una determinada categoria semántica obtenian resultados muy bajos. Así, se concluye que las personas con este tipo de demencia tienen graves problemas para discriminar unidades dentro de una misma categoria, por lo que las parafasias semánticas son constantes.

\footnotetext{
4 Otros modelos, como el de Levelt et al. (1999), sitúan un proceso intermedio entre el semántico o conceptual y el léxico, al que denominan "lemma". En este nivel se encontraría la información gramatical de las palabras: categoría gramatical, género, número, tiempo, modo, persona, etc. Sin embargo, para muchos autores, esta información estaría incluida en el nivel léxico, por lo que no contemplan hacer una distinción en este punto.

${ }^{5}$ En cambio, los problemas de índole léxico-semántica en personas ancianas tendrían como causa un déficit en el acceso léxico, lo que se traduce en una multitud de casos del fenómeno de la "punta de la lengua".
} 
Otras veces, las relaciones entre el objeto nombrado y la palabra que efectivamente se expresa no son nada evidentes. Esta alteración, pocas veces descrita, podemos encontrarla entre las características del discurso de Elsie, la hablante estudiada por Hamilton (1994) durante cinco años. En dos de sus conversaciones, separadas por seis meses, Elsie utiliza el término dress en lugar de picture para referirse a un cuadro que está colgado en la habitación. En la primera conversación, Elsie se da cuenta de que esa no es la palabra que busca y la sustituye por otra, name, que tampoco está relacionada con el término. En la segunda conversación, la investigadora corrige a Elsie, que es capaz de repetir picture, pero inmediatamente después utiliza un término genérico, thing, para referirse al mismo objeto.

El hecho de que esta informante fuera capaz de autocorregirse en la primera conversación y que aceptara una rectificación hecha por su interlocutora en la segunda, nos indica que era consciente de su problema semántico. Esta consciencia del déficit es propia de la primera etapa de la demencia -más tarde se pierde- y uno de sus efectos es el uso de constantes "circunloquios", bien sea para enmascarar su dificultad a la hora de recuperar la palabra o bien porque es el recurso que da un mejor resultado al demente.

A veces los errores semánticos dan lugar a palabras que no significan nada, a puros "neologismos" incomprensibles para las personas que conversan con el hablante demenciado. No ha sido uno de los fenómenos más citados por los estudios. No obstante, Hamilton (1994: 50) afirma que su informante sigue produciendo neologismos hasta etapas avanzadas de la enfermedad. A este respecto, Elsie deja de utilizar bastante pronto los circunloquios y las palabras relacionadas semánticamente. En cambio, los neologismos, los términos genéricos o vacíos y las palabras con un significado no relacionado perduran más tiempo. La explicación para este comportamiento puede estar en que:

In lieu of the intended word, the use of a circumlocution of a semantically related word indicates a greater other-orientation than does the use of a newly coined word, an inexplicit word, or a completely unrelated word (Hamilton, 1994: 50).

Finalmente, la progresión del deterioro semántico alcanza un grado en el que estos sujetos simplemente no responden a la petición de que nombren un objeto o una persona determinados.

\subsubsection{EL DÉFICIT PRAGMÁTICO}

En el caso de las personas con demencia, las entrevistas de tipo terapéutico han sido uno de los mecanismos más empleados para evaluar sus capacidades comunicativas, una vez que el uso de una metodología específicamente de tipo conversacional o discursivo ha sido bastante escaso. Así, por ejemplo, algunos estudios parciales han analizado la falta 
de coherencia y la alta frecuencia de cambios de turno (Ripich y Terrell, 1988) o las dificultades con la gestión temática (Mentis et al., 1995), aunque hay autores que reconocen que las habilidades comunicativas podrían no parecer tan alteradas si las interacciones estudiadas se dieran en un contexto natural y no en situaciones más formales (Moss et al., 2002). El tipo de tareas planteadas y los temas de los que se pide hablar al informante demenciado son, a menudo, poco propicios para que este muestre todas sus habilidades comunicativas.

Dividiremos las habilidades pragmáticas en tres ámbitos o esferas (Gallardo Paúls, 1996): la pragmática enunciativa, la pragmática textual y la pragmática interactiva. Dentro de la pragmática enunciativa, centrada en las categorias especificamente derivadas del hecho enunciativo, como los actos de habla o las inferencias, Hutchinson y Jensen (1980) descubrieron que los sujetos con demencia usaban más actos de habla directivos (peticiones y preguntas) que los individuos sanos con edad avanzada. En cambio, se ha comprobado la presencia de una menor cantidad de actos asertivos, lo que podría significar que estas personas utilizan los actos directivos como recursos para ganar tiempo mientras dan con la respuesta adecuada. En contra del mantenimiento de la capacidad para hacer preguntas está Bayles (1984), quien observa su deterioro en la etapa moderada de la demencia.

Por otra parte, los sujetos con DTA parecen tener problemas desde el principio con el reconocimiento de actos de habla indirectos, ya que, según señalaron Appell et al. (1982), sus informantes no eran capaces de establecer inferencias que les llevaran desde el significado literal al significado figurado o implícito. En muchas ocasiones, este problema puede observarse a partir de respuestas del tipo si/no que estos hablantes dan a preguntas que en realidad son peticiones de más información. Para Hamilton (1994), este problema puede deberse, al igual que otros muchos relacionados con las habilidades pragmáticas, a que la persona con DTA no es capaz de ponerse en lugar de su interlocutor y, por tanto, no entiende por qué emplea un recurso indirecto cuando podría explicarse de forma directa.

Claramente relacionada con esta alteración pragmática se encuentra la dificultad para entender las implicaturas conversacionales. Hays et al. (2004) llevan a cabo un estudio en el que constatan que muchos de los problemas que presentan las personas con DTA en la conversación están relacionados con la transgresión de las máximas conversacionales. Así, la producción de enunciados irrelevantes va en contra, como su propio nombre indica, de la máxima de relevancia. Igualmente, aunque en los primeros momentos de la enfermedad se observe un pequeño aumento en la longitud de los enunciados, a lo largo de la progresión de la demencia los hablantes tienden a hablar cada vez menos. Esta situación normalmente viene acompañada de una disminución en la cantidad de 
información (Hier, et al., 1985), lo que resulta en la violación de la máxima de cantidad, por una parte, y de la máxima de cualidad, por otra.

Dentro de las categorias que incluimos en la pragmática textual se encuentran aquellas que dan forma a las superestructuras textuales (coherencia y cohesión) y la gestión temática. Muchas investigaciones (Obler y Albert, 1984; Müller-y Guendouzi, 2005) dan cuenta de la falta de coherencia que muestran los textos producidos por las personas demenciadas. Para que un discurso resulte coherente debe estar ordenado y mantener el tema de discusión, entre otros aspectos. En los hablantes con DTA la coherencia global se muestra mucho más alterada que la local, es decir, los errores en la superestructura textual vendrian provocados no tanto por las relaciones de significado entre conceptos contiguos en el discurso como por la alteración de la capacidad para organizarlo.

Ripich y Terrell (1988), a partir de un estudio que comparaba las habilidades discursivas de ancianos normales y personas con DTA, demostraron que la cohesión estaba mucho más alterada en el segundo grupo. Este problema, tal y como apuntan otros trabajos (Hamilton, 1994; March et al., 2006) se debe principalmente al constante uso de pronombres sin que se haya mencionado anteriormente a qué refieren. Por último, en cuanto a la gestión del tema de conversación, ya hemos mencionado las glosomanías que caracterizan la etapa más avanzada de la enfermedad, en la que el hablante vuelve una y otra vez a sus temas preferidos. Antes de llegar a este punto, estas personas tienen dificultades con la introducción de un nuevo tema o de información nueva sobre el tema de conversación. La consecuencia de ambas alteraciones es la misma: el empobrecimiento de la capacidad discursiva.

La pragmática interactiva, finalmente, se basa en la naturaleza conversacional del lenguaje, en considerar que todo acto de habla es la respuesta a otro anterior. En muchos casos, el deterioro lingüístico se manifiesta en una pequeña disminución de la iniciativa del habla. Sin embargo, se ha probado que la capacidad para tomar el turno está conservada en la etapa inicial y en la moderada de la demencia (Müller y Guendouzi, 2005). También se han llevado a cabo algunos estudios que comparan la cantidad de turnos y el número de enunciados por turno empleados por sujetos con DTA y sujetos ancianos normales. Los resultados han sido diversos. Por ejemplo, los primeros trabajos sobre este asunto (Hutchinson y Jensen, 1980; Ripich y Terrell, 1988), basados en conversaciones orientadas por los investigadores, recogian que las personas con demencia producian menos enunciados por turno que las personas ancianas, pero a su vez, emitían más turnos que ellos. Sin embargo, en dos estudios de tipo conversacional, Sabat (1991a, b) descubre que los hablantes con DTA y los investigadores producen un número de turnos similar y que donde difieren es en el número de enunciados por turno, que es mucho mayor en las personas con demencia. Esto pudo deberse, no obstante, a que Sabat reconoce que no impuso 
ningún tema en sus conversaciones y que alteró su forma normal de tomar el turno para permitir que sus informantes pudieran hablar todo lo que quisieran. Volvemos a constatar, por tanto, la importancia de la metodología de investigación en Lingüistica clínica.

\subsubsection{El DÉFICIT EN ALGUNAS DESTREZAS SEMIÓTICAS}

Con respecto a la repetición, desde los primeros trabajos que se dedicaron al estudio del lenguaje en las personas con demencia se viene diciendo una y otra vez que se encuentra conservada hasta la última etapa del deterioro lingüístico. Sin embargo, en esta última fase la repetición se produce de forma inconsciente, como reflejo de la pervivencia de las funciones automáticas del lenguaje. El resultado es la presencia de alteraciones que se caracterizan por la repetición de parte del discurso del interlocutor (ecolalia) y por la repetición de unidades o sintagmas producidos por el mismo individuo (perseverancia).

En las DTA la lectura en voz alta está preservada durante gran parte del desarrollo de la enfermedad. Sin embargo, una vez se comprueba el grado de comprensión de lo que han leído, los resultados son mucho peores (Cummings, et al., 1986). Esta disociación demuestra que los problemas en las personas con DTA no son de tipo visual, sino que el déficit se encuentra en el procesamiento semántico. Por otro lado, parece ser que la habilidad para la escritura está más deteriorada que la lectura. Algunos autores, como Rapcsak et al. (1989), propusieron que los fallos en la escritura podrian servir para observar la evolución de la demencia, ya que las alteraciones gráficas van en consonancia con la gravedad del deterioro cognitivo. Así, al principio las alteraciones en la escritura estarian relacionadas con una reducción en la cantidad de palabras y en la complejidad de las oraciones que producen en una tarea de redacción libre. Groves et al. (2004) también descubrieron alteraciones a nivel léxico (sustitución de palabras de contenido, omisiones y perseveraciones). Finalmente, la escritura se simplifica aún más y se producen alteraciones en el orden de las grafias hasta que se llega a un punto en el que el sujeto es incapaz de escribir nada.

\section{Conclusiones}

Con este trabajo hemos pretendido establecer una panorámica de los estudios que analizan el deterioro lingüístico en personas con la enfermedad de Alzheimer. Nos parece que la especificidad de la mayoría de estas investigaciones hacía necesario un trabajo recopilatorio en el que se recogiesen las principales aportaciones que se han hecho al tema de estudio desde diversas disciplinas científicas y con diferentes métodos de investigación. Con respecto a este último asunto, nuestra postura se sitúa junto a la de aquellos que abogan por el uso de datos recogidos en 
contextos naturales pese a que, como hemos podido comprobar, son mayoria los trabajos que no consideran la validez ecológica de los datos.

El repaso de la bibliografia existente sobre déficit lingüístico en DTA nos demuestra la importancia de evaluar el lenguaje de estas personas a la hora de diagnosticar la enfermedad y de establecer diferentes etapas. Del mismo modo, cabe esperar que el análisis de los componentes lingüísticos más deteriorados (en este caso, el nivel léxico-semántico y el pragmático) nos permita crear protocolos de conversación con los que ayudar a mejorar la calidad de vida de los pacientes y de sus familias.

\section{REFERENCIAS}

ALONSO, D., CHAMARRO, R. \& SANTONJA, J. M. (2004): "Trastornos del lenguaje y demencia. Experiencia de nuestra Unidad de Demencias", Gallardo Paúls, B. y Veyrat Rigat, M. (eds.): Lingüistica y patologia, Valencia: Universitat de València, pp. 5-30.

APPELL, J., KERTESZ, A. \& FISMAN, M. (1982): "A study of language functioning in Alzheimer patients", Brain and Language, 17, pp. 73-91.

BATES, E., HARRIS, C., MARCHMAN, V., WULFECK, B. \& KRITCHEVSKY, M. (1995): "Production of complex syntax in normal aging and Alzheimer's disease", Language and Cognitive Processes, 10, pp. 487-539.

BAYLES, K. (1984): "Language and dementia", Holland, A. (ed.): Language disorders in Adults: Recent Advances, San Diego: College-Hill Press, pp. 209-244.

BAYLES, K. \& TOMOEDA, Ch. (1990): "Naming and Categorial Knowledge in Alzheimer's Disease: The Process of Semantic Memory Deterioration", Brain and Language, 39, pp. 498-510.

BAYLES, K. \& TOMOEDA, Ch. (1996): Functional Linguistic Communication Battery, Tucson: Canyonlands Publishing.

BLAIR, M., MARCZINSKI, C., DAVISFAROQUE, N. \& KERTESZ, A.
(2007): "A longitudinal study of language decline in Alzheimer's disease and frontotemporal dementia", Journal of the International Neuropsychological Society, 13, pp. 237-245.

CHAPMAN, S. \& ULATOWSKA, H. (1997): "Discourse in dementia: Considerations of consciousness", Stamenov, M. (ed.): Language structure, discourse and the access to consciousness, Amsterdam: John Benjamins, pp. 155-188.

CROOT, K., HODGES, J., XUEREB, J. \& PATTERSON, K. (2000): "Phonological and Articulatory Impairment in Alzheimer's Disease: A Case Series", Brain and Language, 75, pp. 277-309.

CUETOS, F. (2003): Anomia: La dificultad para recordar las palabras, Madrid: TEA.

CUMMINGS, J., HOULIHAN, J. \& HILL, M. (1986): "The Pattern of Reading Deterioration in Dementia of the Alzheimer Type: Observations and Implications", Brain and Language, 29, pp. 315323.

EMERY, O. (1985): "Language and aging", Experimental Aging Research, 11 (1), pp. 3-60.

FERRIS, S., IHL, R., ROBERT, P., WINBLAD, B., GATZ, G., TENNIGKEIT, F. \& GAUTHIER, S. (2009): "Severe Impairment Battery Language scale: A 
language-assessment tool for Alzheimer's disease patients", Alzheimer's \& Dementia, 5, pp. 375-379.

FOLSTEIN, M., FOLSTEIN, S. \& McHUGH, P. (1975): "Mini-mental state. A practical method for grading the cognitive state of patients for the clinician", Journal of psychiatric research, 12 (3), pp. 189-198.

FORBES-McKAY, K. \& VENNERI, A. (2005): "Detecting subtle spontaneous language decline in early Alzheimer's disease with a picture description task", Journal of the Neurological Sciences, 26, pp. 243-254.

GALLARDO PAÚLS, B. (1996): Análisis conversacional $y$ pragmática del receptor, Valencia: Episteme.

<www.uv.es/pauls/TODO.PDF> [13/04/12]

GALLARDO PAÚLS, B. (2006): "Más allá de las palabras y la estructura: las categorias del componente pragmático", Garayzábal, E. (coord.): Lingüistica clinica y logopedia, Madrid: Antonio Machado Libros, pp. 81-196.

GLOSSER, G., KOHN, S., FRIEDMAN, R., SANDS, L. \& GRUGAN, P. (1997): "Repetition of single words and nonwords in Alzheimer's disease", Cortex, 33, pp. 653-666.

GROVES-WRIGHT, K., NEILSSTRUNJAS, J., BURNETT, R. \& O'NEILL, M.J. (2004): “A comparison of verbal and written language in Alzheimer's disease", Journal of Communication Disorders, 37, pp. 109-130.

HAMILTON, H. (1994): Conversations with an Alzheimer's Patient, Cambridge: Cambridge University Press.

HAYS, S., NIVEN, B., GIDFREY, H. \& LINSCOTT, R. (2004): "Clinical
Assessment of Pragmatic Language Impairment: A generalisability study of older people with Alzheimer disease", Aphasiology, 18 (8), pp. 693-714.

HIER, D., HAGENLOCKER, K. \& SCHINDLER, A. (1985): "Language disintegration in dementia: effects of etiology and severity", Brain and Language, 25, pp. 117-133.

HORNER, J., DAWSON, D., HEYMAN, A. \& McGORMAN, A. (1992): "The Usefulness of the Western Aphasia Battery for Differential Diagnosis of Alzheimer Dementia and Focal Stroke Syndromes: Preliminary Evidence", Brain and Language, 42, pp. 77-88.

HUFF, J., CORKIN, S. \& GROWDON, J. (1986): "Semantic Impairment and Anomia in Alzheimer's Disease", Brain and Language, 28, pp. 235-249.

HUTCHINSON, J. \& JENSEN, M. (1980): "A pragmatic evaluation of discourse communication in normal and senile elderly in a nursing home", Obler, L. \& Albert, M. (eds.): Language and Communication in the Elderly, Lexingtong: Lexingtong Books, pp. 59-73.

LABOS, L. E. (2002): Alteraciones de la producción lingüística en las demencias tipo Alzheimer, Tesis de doctorado, Universidad de Buenos Aires.

LEVELT, W., ROELOFS, A. \& MEYERS, A. (1999): "A theory of lexical access in speech production", Behavioral and Brain Sciences, 22, pp. 1-75.

MACLAGAN, M. \& MASON, P. (2005): "Bad Times and Good Times: Lexical Variation over Time in Robbie Walters' Speech", Davis, B. (coord.): Alzheimer Talk, Text and Context: Enhancing Communication, New York: Palgrave Macmillan, pp. 146-166. 
MACOIR, J. \& TURGEON, Y. (2006): "Dementia and language", Brown, K. (ed.): The Encyclopedia of Language and Linguistics, Oxford: Elsevier, pp. 423-430.

MARCH, E., WALES, R. \& PATTISON, P. (2006): "The uses of nouns and deixis in discourse production in Alzheimer's disease", Journal of Neurolinguistics, 19, pp. 311-340.

MARTIN, A. \& FEDIO, P. (1983): "Word Production and Comprehension in Alzheimer's Disease: The Breakdown of Semantic Knowledge", Brain and Language, 19, pp. 124-141.

MENTIS, M., BRIGGS-WHITTAKER, J. \& GRAMIGNA， G. (1995): "Discourse topic management in senile dementia of the Alzheimer's type", Journal of Speech and Hearing Research, 38, pp. 10541066.

MOSS, S., POLIGNANO, E., WHITE, C., MINICHIELLO, M. \& SUNDERLAND, T. (2002): "Reminiscence group activities and discourse interaction in Alzheimer's disease", Journal of Gerontological Nursing, 28, pp. 2644.

MÜLLER, N. \& GUENDOUZI, J. (2005): "Order and disorder in conversation: Encounters with dementia of the Alzheimer's type", Clinical Linguistics and Phonetics, 19 (5), pp. 393-404.

MUÑOZ CÉSPEDES, J.M. \& MELLE, N. (2004): "Alteraciones de la pragmática de la comunicación después de un traumatismo craneoencefálico", Revista de Neurologia, 38 (9), pp. 852-859.

MURDOCH, B., CHENERY, H., WILKS, V. \& BOYLE, R. (1987): "Language Disorders in Dementia of the Alzheimer Type", Brain and Language, 31, pp. 122-137.

MURILlO, B. (1999): Estudio de la evolución del lenguaje en la demencia tipo Alzheimer,
Barcelona: Instituto Superior de Estudios Psicológicos.

MURRAY, L. (2010): "Distinguishing clinical depression from early Alzheimer's disease in elderly people: Can narrative analysis help?", Aphasiology, 24, pp. 928939.

NAKAMURA, H., NAKANISHI, M., HAMANAKA, T., NAKAAKI, S. \& YOSHIDA, S. (2000): "Semantic priming in patients with Alzheimer and semantic dementia", Cortex, 36, pp. 151162.

OBLER, L. \& ALBERT, M. (1984): "Language in aging", Albert, $\mathrm{M}$. (ed.): Clinical Neurology of Aging, New York: Oxford University Press, pp. 245-253.

PAGANELLI, F., VIGLIOCCO, G., VINSON, D., SIRI, S. \& CAPPA, S. (2003): "An investigation of semantic errors in unimpaired and Alzheimer's speakers of Italian", Cortex, 39, pp. 419-439.

PRICE, B., GURVIT, H., WEINTRAUB, S., GEULA, C., LEIMKUHLER, E. \& MESULAM, M. (1993): "Neuropsychological patterns and language deficits in 20 consecutive cases of autopsyconfirmed Alzheimer's disease", Archives of Neurology, 50, pp. 931-937.

RAMANATHAN, V. (1997): Alzheimer discourse: some sociolinguistic aspects, Mahwah (NJ): Lawrence Erlbaum.

RAPCSAK, S., ARTHUR, S., BLINKEN, D. \& RUBENS, A. (1989): "Lexical agraphia in Alzheimer's disease", Archives of Neurology, 46, pp. 66-68.

Rhys, C. \& SChMidT-Renfreee, N. (2000): "Facework, social politeness and the Alzheimer's patient", Clinical Linguistics and Phonetics, 14 (7), pp. 533-543.

RIPICH, D. \& TERRELL, B. (1988): "Patterns of discourse cohesion 
and coherence in Alzheimer's disease", Journal of Speech and Hearing Disorders, 53, pp. 8-15.

ROBLES BAYÓN, A. \& VILARIÑO, I. (1998): "Trastornos del lenguaje en la demencia", Juncos, O. (coord.): Lenguaje $y$ Envejecimiento. Bases para la intervención, Barcelona: Masson, pp. 73-97.

ROCHON, E., WATERS, G. \& CAPLAN, D. (1994): "Sentence Comprehension in Patients with Alzheimer's Disease", Brain and Language, 46, pp. 329-349.

ROSEN, W., MOSH, R. \& DAVIS, K. (1984): "A new rating scale for Alzheimer's disease", American Journal of Psychiatry, 141 (11), pp. 1356-1364.

SABAT, S. (1991a): “Turn-taking, turn-giving, and Alzheimer's disease: a case study of conversation", The Georgetown
Journal of Language and Linguistics, 2, pp. 167-181.

SABAT, S. (1991b): "Facilitating conversations via indirect repair: a case study of Alzheimer's disease", The Georgetown Journal of Language and Linguistics, 2, pp. 284-296.

SCHRÖDER, J., WENDELSTEIN, B. \& FELDER, E. (2010): "Language in the preclinical stage of Alzheimer's disease: Content and complexity in biographical narratives", Alzheimer's \& Dementia, 6 (4) (Supl.), pp. 360361.

SMITH, S., FAUST, M., BEEMAN, M. KENNEDY, L. \& PERRY, D. (1995): "A Property Level Analysis of Lexical Semantic Representation in Alzheimer's Disease", Brain and Language, 49, pp. 263-279. 